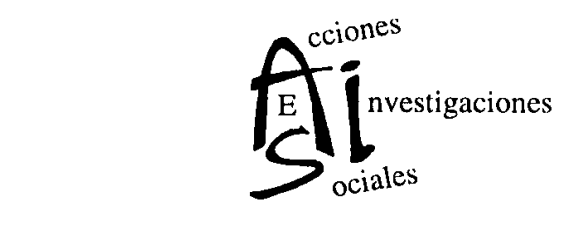

\title{
La importancia creciente del voluntariado
}

\author{
Sonia Isabel Pedrosa Alquézar \\ DEPARTAMENTO DE DERECHO DE LA EMPRESA \\ UNIVERSIDAD DE ZARAGOZA
}

\begin{abstract}
El voluntariado puede definirse como el conjunto de actividades de interés general desarrolladas por personas físicas, siempre que las mismas no se realicen en virtud de una relación laboral, funcionarial, mercantil o cualquier otra retribuida, sin perjuicio de que complementen la actividad de estas otras relaciones. Si bien, las personas voluntarias, en el desarrollo de esas actividades, tienen una serie de obligaciones que se asemejan a las derivadas de un contrato de trabajo. Esto hace pensar en si no sería conveniente ampliar la protección social de los voluntarios y, a su vez, pone de manifiesto que las áreas cubiertas por el voluntariado pueden convertirse en yacimientos de empleo que generarán nuevos puestos de trabajo.
\end{abstract}

Palabras clave: Voluntariado, relación laboral, protección social, empleo, Tercer Sector, trabajo. 


\section{La importancia creciente del voluntariado}

Sonia Isabel Pedrosa Alquézar

\section{Introducción}

La idea de trabajo está cambiando. El criterio principal de identificación del contrato de trabajo, la subordinación, evoluciona en dos direcciones. Por una parte, hacia una concepción mucho más relajada de las relaciones empleador-empleado. Esto supone una mayor flexibilidad y responsabilidad del trabajador en la ejecución del trabajo. Por otra, se hace más fuerte por la precarización del trabajo y la existencia de un paro masivo que lleva a los trabajadores a someterse a condiciones laborales que rechazarían si encontraran fácilmente otro empleo. Evolucionan también las formas de organización del trabajo (externalización, deslocalización...) y aparece con ellas una nueva concepción del tiempo en el mismo, heterogéneo e individualizado. Además, la negociación colectiva se va convirtiendo en la principal norma reguladora de las relaciones de trabajo, negociación que debe adaptarse a las nuevas formas de organización del mismo.

Por otro lado, dentro de esta evolución, el desempleo ha dejado de ser una situación ocasional, de corta duración, para convertirse en un mal endémico y uno de los principales problemas del mercado de trabajo al que debe hacerse frente'.

1 Vid.para todos estos cambios:VVAA (1998): Cambios en el trabajo y futuro del Derecho del Trabajo en Europa. Informe (Versión provisional).Grupo de ex pertos creado por la DG.V de la Comisión Europea. Luxemburgo. Oficina de Publicaciones de la Unión Europea. 
Tanto en España como en la Unión Europea se están realizando propuestas y adoptando iniciativas para intentar reducir este desempleo o, al menos, sus efectos. Así, en nuestro país se ha intentado fomentar la contratación indefinida con bonificaciones en las cuotas a la Seguridad Social y reducciones en algunas indemnizaciones por despido²; fomentar la formación y la contratación fija a tiempo parcial; disminuir las horas extraordinarias y compensarlas por tiempo de descanso...

Por su parte, la Unión Europea, consciente del problema del desempleo, considera como uno de sus objetivos la realización de una adecuada política de empleo ${ }^{3}$. Así, en las Conclusiones de la Presidencia del Consejo Europeo Extraordinario sobre el empleo celebrado en Luxemburgo los días 20 y 21 de noviembre de $1997^{4}$ se establecieron una serie de directrices con medidas a adoptar por los Estados miembros para conseguir una eficaz política de empleo:

- Mejorar la capacidad de inserción profesional combatiendo el desempleo juvenil, previniendo el desempleo de larga duración, sustituyendo medidas pasivas por medidas activas, fomentando un planteamiento de cooperación y facilitando la transición de la escuela a la vida laboral.

- Fomentar la capacidad de adaptación de los trabajadores y de las empresas modernizando la organización del trabajo y apoyando la capacidad de adaptación de esas empresas.

- Reforzar la política de igualdad de oportunidades combatiendo la discriminación entre hombres y mujeres, conciliando la vida laboral con la vida familiar, facilitando la rein-

2 Vid.entre otros, por la relevancia que tuvieron, Real Decreto-ley 8/1997, de 16 de mayo, de Medidas Urgentes para la mejora del mercado de trabajo y el fomento de la contratación indefinida (BOE núm 118 de 17 de mayo) y Real Decreto-Ley 9/1997, de 16 de mayo, por el que se regulan incentivos en materia de Seguridad Social y de carácter fiscal para el fomento de la contratación indefinida y la estabilidad en el empleo (BOE núm 118 de 17 de mayo).

3 Vid. Tratado de Amsterdam por el que se modifica el Tratado de la Unión Europea, los Tratados Constitutivos de las Comunidades Europeas y determinados actos conexos, firmado el 2 de Octubre de 1997 (DOCE № C 340 de 10 de noviembre de 1997).

4 VVAA (1998): Conclusiones de la Presidencia del Consejo Europeo Extraordinario sobre el empleo celebrado en Luxemburgo los días 20 y 21 de no viembre de 1997. Luxemburgo: Oficina de Publicaciones de la Unión Europea. 
corporación al trabajo y favoreciendo la inserción de los minusválidos en el mismo.

- Desarrollar el espíritu de empresa facilitando la creación y gestión de empresas, adaptando el régimen fiscal para hacerlo más favorable al empleo y aprovechando las oportunidades de creación de puestos de trabajo. Una de estas oportunidades la ofrece la Economía Social o también denominada Tercer Sector.

El objeto de este trabajo es, fundamentalmente, determinar en que medida las actividades que se desarrollan en ese Tercer Sector pueden convertirse en nuevas formas de trabajo que ayuden a crear empleo.

\section{Trabajo voluntario: necesidad de mayor protección social y descubrimiento de nuevas formas de trabajo}

La Economía Social o el Tercer Sector son las organizaciones de la sociedad civil que no responden a la lógica del funcionamiento del sector público-estatal ni a la del sector privado mercantil-empresarial ${ }^{5}$.

Estas organizaciones se caracterizan por su independencia frente al Gobierno, no tener fin de lucro, autogobernarse, articular valores de cambio social, detectar necesidades de bienestar social e instrumentar respuestas para su satisfacción; en definitiva, por favorecer la integración social y la consolidación de una sociedad pluralista. Para conseguir este objetivo sus funciones fundamentales son, entre otras, las siguientes ${ }^{6}$ : actuar como agentes innovadores dada su flexibilidad en los enfoques de los problemas, la profundidad en los métodos utilizados y la libertad en la toma de decisiones con los que se han dado respuestas nuevas y originales a problemas particularmente difíciles; prestar servicios para llegar allí donde no llegan los servicios públi-

5 Vid, en este sentido.:Coduras, P.(1995): «Voluntarios, discípulos y ciudadanos».Cuadernos de Cristianismo y Justicia, (p. 11).

6 Vid.Salinas Ramos, F (1997): «El estado de bienestar y las organizaciones voluntarias» en VVAA.: ¿Trabajo voluntario o participación?.Elementos para una sociología del Tercer Sector. Madrid: Tecnos. (p. 124). 
$\cos ^{7}$ y complementar las labores de éstos allí donde existan pero sean insuficientes; actuar como defensores de los intereses de la sociedad y aspirar a una constante transformación social en beneficio de todos los ciudadanos; preservar alguno de los valores de nuestra sociedad como la participación, el pluralismo, y la democracia...; fomentar nuevos valores y nuevos principios; mediar entre las instituciones de la vida privada y de la vida pública etc... Para la realización efectiva de todas estas funciones necesitan apoyo estatal (financiación ${ }^{8}$, utilización de locales públicos...) y, en algunas ocasiones, apoyo del sector privado ; por eso puede decirse que los tres sectores deben interrelacionarse para conseguir un efectivo bienestar social.

Esas organizaciones del Tercer Sector pueden ser religiosas; relacionadas con el trabajo (como sindicatos y organizaciones profesionales);relacionadas con la instrucción (escuelas de todo tipo y organizaciones de investigación, entre otras); fundaciones (como algunas ONGDs);organizaciones culturales (bibliotecas y museos, entre otros); de servicios (por ejemplo, aquellas que se dedican a prestar servicios sociales); organizaciones sociales (como las deportivas o étnicas); las relacionadas con la sanidad (asociaciones que se ocupan de enfermedades específicas, por ejemplo); de caridad; políticas y movimientos de masas organizados. ${ }^{10}$

Este Tercer Sector se denomina también Economía Social en la medida en que muchas de estas Organizaciones buscan la

7 Incluso, a veces, estas organizaciones han venido prestando servicios para hacer frente a determinadas situaciones sociales antes de que la Administración contemplara algún tipo de respuesta (Vid. Exposición de Motivos de la Ley 17/1998, de 25 de junio, del voluntariado social del País Vasco - BOPV núm.130, de 13 de julio- y Exposición de Motivos de la Ley Foral 2/1998, de 27 de marzo, del voluntariado en la Comunidad Foral de Navarra (BOE núm 131 de 2 de junio).

8 Su autonomía respecto al sector público y privado supone que esa financiación la obtienen, también, del apoyo económico de sus colaboradores o por venta de materiales, colecta de fondos, realización de cursos...

9 Ejm. Empresa Ram en colaboración con Unicef o Empresa Aixertell que destinó en la Navidad de 1997 parte de los beneficios obtenidos por la compra de sus productos a un proyecto de desarrollo integral en Ecuador promovido por la ONGD Ayuda en Acción.

10 Ascoli, H.(1987): «Estado de bienestar y acción voluntaria». Revista Española de Investigaciones Sociológicas, 38, (pp. 123-124). 
solución de problemas sociales con criterios de rentabilidad y eficacia empresarial ${ }^{11}$.

Además de contar con personal contratado laboralmente, es decir, a través de una relación jurídica en la que se prestan servicios retribuidos por cuenta de la Organización y dentro del ámbito de dirección de la misma, generalmente para desempeñar tareas básicas de funcionamiento, esas Organizaciones del Tercer Sector actúan con voluntarios.

Voluntario es aquella persona que, desde la gratuidad, se compromete a dedicar parte de su tiempo libre ${ }^{12}$ a trabajar en actividades de interés general, entendiendo por tales las asistenciales, de servicios sociales, cívicas, educativas, culturales, científicas, deportivas, sanitarias, de cooperación al desarrollo, de defensa del medio ambiente o cualquier otra de naturaleza análoga ${ }^{13}$.

El derecho a ser voluntario no viene recogido como tal en nuestra Constitución, si bien puede entenderse reconocido en el derecho de todos los ciudadanos a participar en la vida económica, política, cultural y social (art. 9.2 CE), así como en los distintos artículos que aluden a los poderes públicos como comprometidos con diversas acciones en las que el voluntario participa. Así, el art. 49 CE (previsión, tratamiento, rehabilitación e integración de los disminuidos físicos, sensoriales y psíquicos);el $50 \mathrm{CE}$ (búsqueda de bienestar de los ciudadanos de la tercera edad mediante un sistema de servicios sociales que atenderá sus problemas específicos de salud, vivienda, cultura y ocio);el $43.2 \mathrm{CE}$ (protección a la salud), el 39 CE (protección a la familia y a la infancia); el 45 CE (protección al medio ambiente)...

Ser voluntario implica compromiso y decisión libre y responsable, actuación desde un programa de acción establecido por la or-

11 Mogin Barquín, M.T.(1997): «Provisión privada-social y gestión mixta de servicios públicos». Revista de Debate sobre Economía Pública Social y Cooperativa , 26, (p.13). Si bien, debe advertirse que, en determinadas ocasiones, el término Economía Social hace referencia a tres grupos específicos de organizaciones: Cooperativas, Sociedades Laborales y Empresas de Inserción (Ibidem, pp (13 y 14).Vid.también:Vidal, I (1997): «España: Una nueva economía social aún poco conocida y reconocida» en VVAA: Inserción y nueva Economía Social (pp. 236-250).Madrid:CIRIEC.).

12 En consecuencia, los voluntarios también deben tener una jornada de trabajo.

13 Vid, art. 4 de la Ley estatal 6/1996 de 15 de enero del voluntariado social (BOE núm 15, de 17 de Enero) 
ganización ${ }^{14}$, trabajo o, si entendemos por tal, estrictamente, una actividad que produce bienes y servicios de valor económico mensurable para otros y no cualquier actividad de utilidad social, actividad $^{15}$ altruista ${ }^{16}$ solidaria y no remunerada ${ }^{17}$. En consecuencia, esta actividad voluntaria nunca podría ser considerada una relación jurídica laboral puesto que le falta una de sus principales notas definitorias, la retribución. Así lo establecen, expresamente, además de recoger las otras características mencionadas, las diversas leyes sobre la relación jurídica del voluntariado que existen en nuestro país ${ }^{18}$.Así, por ejemplo, la Ley estatal 6/1996, de 15 de enero, reguladora del voluntariado social lo define, en su artículo 3 , como «el conjunto de actividades de interés general, desarro -

14 Así, no se consideran actividades voluntarias a efectos jurídicos aquellas actividades esporádicas realizadas por razones de amistad, benevolencia 0 buena vecindad.

15 Vid, en este sentido, Le Guidec, R.(1996): iDecadencia y resurgimiento del trabajo gratuito".Revista_Internacional del Trabajo, vol.115, 6, (pp.23-34).

16 Renes, V; Alfaro, E ; Ricciardelli, O.(1994): El voluntariado social. Plan de formación de animadores. Bloque I. Madrid:CES, (p. 34).Vid.también, Bernardo, F y Renes, V.(1986): «Voluntariado» en Materiales para un glosario sobre Bienestar Social. Madrid:Cáritas/CEBS.

17 Sin perjuicio de que el voluntario reciba una compensación económica por los gastos realizados en el desempeño de su actividad (Vid. art. 6.d) de la Ley Estatal 6/1996)

18 No obstante, no deben olvidarse como documentos fundamentales en el impulso y desarrollo del voluntariado la Carta Europea de los Voluntarios y la Recomendación no 89 de 21 de Junio de 1985 del Comité de Ministros del Consejo de Europa. La primera representa una declaración de intenciones. Establece que cualquier definición de voluntario debe incluir, al menos, los siguientes elementos: las actividades se ocupan de otras personas o de la sociedad; carece de interés económico personal; se desarrolla en un marco más o menos organizado; es una expresión libre y expresa por medios pacíficos. Además establece que todo trabajo voluntario es valioso para la comunidad, que todo ciudadano tiene el derecho (que no la obligación) a realizar un trabajo voluntario de acuerdo con sus capacidades y enuncia una serie de derechos y obligaciones para los voluntarios.Por otro lado, distingue el voluntariado del trabajador retr ibuido. Comenta que cada uno de ellos debe reconocer el valor de la contribución del otro, y cada uno debe recibir un reconocimiento por parte de la sociedad y por parte de la asociación para la que trabajan en cooperación. Por su parte, la segunda recomienda a los gobiernos de los Estados miembros que reconozcan el papel, las características y el valor del trabajo voluntario realizado de manera desinteresada por personas que por su propia voluntad participan en la acción social, y tomen las medidas apropiadas a fin de definir y mejorar las modalidades de realización de tal trabajo según los principios que ella misma establece. 
lladas por personas físicas, siempre que las mismas no se reali cen en virtud de una relación laboral, funcionarial, mercantil o cualquier otra retribuida», sin perjuicio de que complementen la actividad de estas otras relacione ${ }^{19}$, se realicen en las debidas condiciones de seguridad y salud y se proteja al voluntario contra los riesgos de enfermedad y accidente derivados del ejercicio de la actividad voluntaria. Las normas autonómicas sobre voluntariado también vienen a definirlo de esta forma ${ }^{20}$.

Si bien, a pesar de ser una actividad no remunerada, estos voluntarios y las Entidades en las que colaboran tienen una serie de obligaciones que se asimilan a algunas de las derivadas de un contrato de trabajo. Así, entre otras, la Organización viene obligada a velar por el respeto de las funciones asignadas a cada uno de los miembros de los diferentes proyectos y programas; a informar y a orientar adecuadamente a las personas voluntarias que colaboren en ella sobre su organización y funcionamiento y sobre las actividades que en ésta hayan de realizar aquéllos; a dotar a las personas voluntarias de los medios adecuados para el cumplimiento de sus cometidos; a responsabilizarse de los daños y perjuicios causados por los voluntarios a terceras personas, a garantizar la seguridad y salud en el trabajo y a la suscripción de un seguro de accidentes y enfermedad. Respecto a este seguro y al de responsabilidad hacia terceros, debe advertirse que, para suscribirlo, las Organizaciones, con base en la continuidad que requiere la actividad del voluntariado, exigen una dedicación ${ }^{21}$ semanal, generalmente de seis a diez horas, al proyecto al que son asignados.

19 En este sentido debe advertirse, como pone de manifiesto la Exposición de Motivos de la Ley 17/1998, de 25 de junio, del Voluntariado Social del País Vasco citada, que no debe entenderse la acción social voluntaria como un sucedáneo de la actividad profesional, vía para hacer dejación de responsabilidades a la hora de crear servicios públicos que respondan a demandas sociales.

20 Entre esas normas autonómicas pueden destacarse: Ley Foral 2/1998, de 27 de marzo, del voluntariado en la Comunidad Foral de Navarra, ya citada supra;Ley 7/1998, de 6 de mayo, del voluntariado en la Comunidad Autónoma de la Rioja (BOE núm 125 de 26 de mayo); Ley 4/1998, de 15 de mayo, de voluntariado de Canarias (BOE núm 134 de 5 de junio); Ley 7/2001, de 12 de julio del voluntariado de la Comunidad Autónoma de Andalucía (BOE núm.188, de 7 de agosto)

21 Vid. Capítulo III Ley Foral Ley Foral 2/1998, de 27 de marzo, del voluntariado en la Comunidad Foral de Navarra;-Capítulo II Ley 7/1998, de 6 de mayo, del voluntariado en la Comunidad Autónoma de la Rioja y art.9 de la Ley Estatal 6/1996, de 15 de enero, del voluntariado social. 
Por su parte, los voluntarios deben, por un lado, entre otras obligaciones, desarrollar sus actividades con la máxima diligencia y conforme a las exigencias del principio de buena fe, en los términos del compromiso aceptado y de las indicaciones que para el cumplimiento de las mismas pudieran recibir de la entidad en que colaboren, que viene a identificarse con el deber de obediencia, diligencia en la ejecución de trabajo y deber de buena fe contractual; por otro, mantener la confidencialidad de la información recibida y conocida en el desarrollo de su actividad, que viene también a identificarse con ese deber de buena fe contractual.

Esta cierta semejanza con algunas de las obligaciones derivadas de un contrato de trabajo unida a la importancia cada vez más creciente de las actividades de voluntariado hace pensar en la conveniencia de ampliar la protección social de los voluntarios en la realización de sus funciones; una protección que podría ser igual a la que hasta el Real Decreto Ley 8/1997, de 16 de mayo, de Medidas Urgentes para la mejora del mercado de trabajo y el fomento de la contratación indefinida ${ }^{22}$ tenía el contrato a tiempo parcial realizado para menos de 12 horas a la semana o 48 horas al mes que sólo abarcaba las contingencias de accidentes de trabajo y enfermedades profesionales, asistencia sanitaria por contingencias comunes, Fondo de Garantía Salarial y la prestación económica correspondiente a los descansos por maternidad.

A pesar de estas características y la necesidad de ampliación de su status jurídico con algunas de las normas de Seguridad Social aplicables a un contrato de trabajo, la actividad voluntaria, como se ha afirmado, nunca podría llegar a considerarse una relación laboral puesto que le falta una de sus características principales, la contraprestación económica y, por otro lado, la actividad voluntaria no es un instrumento de beneficio económico, sino, como ya se ha dicho, una actividad de utilidad social.

Si bien, pese a las afirmaciones anteriores, podría decirse que el voluntariado es una nueva forma de trabajo o potencial forma de trabajo en la medida en que con el mismo se han des-

22 Disposición Transitoria 4르.

23 Los voluntarios pueden llegar a crear puestos de trabajo en la medida en que "cubren el espacio intermedio entre el surgimiento de las necesidades y la satisfacción de las mismas por los profesionales» (González Blasco, P y 
cubierto ${ }^{23}$ y se van descubriendo nuevos campos, nuevas posibilidades, nuevos yacimientos de empleo ${ }^{24}$ donde más pronto 0 más tarde se va a necesitar un contingente de trabajadores para coordinar, administrar y ejecutar las tareas que surgen de esas posibilidades. Con el fomento de estos yacimientos se consigue crear empleo y contribuir a la disminución del paro ${ }^{25}$.

\section{Conclusiones}

En la actualidad, el desempleo ha dejado de ser una situación ocasional para convertirse en uno de los principales problemas del mercado de trabajo. Para hacerle frente, nuestro país y la Comunidad Europea han realizado propuestas y adoptado distintas medidas. Entre estas propuestas se encuentra la de aprovechar las oportunidades de creación de puestos de trabajo que ofrece el Tercer Sector.

Las actividades desarrolladas por el Tercer Sector suelen ser realizadas por personas voluntarias, es decir, por personas que,

Gutiérrez Resa, A (1997): La opinión pública ante el voluntariado. Madrid, Fundación Universidad-Empresa - p 44-). En este sentido, Liebje Hoekendijk afirma que «los voluntarios siempre han creado trabajo remunerado mediante el descubrimiento de nuevos campos, donde, más pronto o más tarde, se retribuyen ciertos puestos, para coordinar, administrar o para otras tareas. Esto sigue ocurriendo aún, a pesar de que en los últimos tiempos ha sido más difícil. Los sindicatos debieran contemplar a los voluntarios como creadores de empleo y no como ladrones. Esto es en interés de los consumidores, voluntarios, de la creación de trabajos retribuidos y de los profesionales»( En Hoekendijk, L.(1987): ¿Qué trabajo debe pagarse?.Madrid.Cruz Roja Española -p 42-).

24 Pueden definirse como actividades destinadas a satisfacer nuevas necesidades sociales que se configuran hoy en mercados incompletos y que tienen un ámbito de producción / prestación territorialmente definido, al mismo tiempo que son intensivos en empleo. Así, acompañamiento de personas de edad al exterior, atención a niños con problemas o restauración (Jiménez, E et alt.(1998): Los nuevos yacimientos de empleo. Barcelona.Fundación CIREM.-pp 43-47-).

25 Además, debe advertirse que, a veces, esas nuevas posibilidades, no quedan en simples experiencias de empleo sino que llegan a ser unas empresas fuertes y competitivas. (Vid, en este sentido Fernández Guerrero, R y Martínez Moreno, A (1997) : «Las empresas de Economía Social en la gestión de servicios de apoyo a personas», Revista de Debate sobre Economía Pública Social y Cooperativa, 26, 1997, -pp 43 -72-). 
desde la gratuidad, se comprometen a lleva a cabo libre y solidariamente esas actividades dentro de un programa establecido.

Este voluntariado aunque tenga obligaciones muy parecidas a las de una relación laboral e incluso se considere conveniente la ampliación de su régimen jurídico con normas de Seguridad Social, no podría considerarse como tal puesto que es una actividad de utilidad social y, además, no está remunerada.

Ahora bien, esa no identificación con la relación laboral no excluye poder considerarla fuente de empleo en la medida en que a través de ella se van descubriendo nuevos yacimientos de empleo que generarán nuevos puestos de trabajo. En definitiva, puede decirse que el voluntariado cubre el espacio intermedio entre el surgimiento de necesidades y la satisfacción de las mismas por los profesionales.

\section{Referencias bibliográficas}

ASCOLI, H.(1987). «Estado de bienestar y acción voluntaria». Revista Española de Investigaciones Sociológicas, 38. (pp. 123-129).

BERNARDO, F y RENES, V.(1986). «Voluntariado» en Materiales para un glosario sobre Bienestar Social. Madrid: Cáritas/CEBS.

CODURAS, P. (1995). «Voluntarios, discípulos y ciudadanos». Cuadernos de Cristianismo y Justicia.

FERNÁNDEZ GUERRERO, R y MARTÍNEZ MORENO, A (1997). «Las empresas de Economía Social en la gestión de servicios de apoyo a personas». Revista de Debate sobre Economía Pública Social y Cooperativa, 26, 1997, (pp 43 -72).

GONZÁLEZ BLASCO, P. y GUTIÉRREZ RESA, A (1997). La opinión pública ante el voluntariado. Madrid, Fundación Universidad-Empresa.

HOEKENDIJK, L. (1987): ¿Qué trabajo debe pagarse?. Madrid. Cruz Roja Española.

JIMÉNEZ, E et al. (1998). Los nuevos yacimientos de empleo. Barcelona. Fundación CIREM. 
LE GUIDEC, R.(1996). «Decadencia y resurgimiento del trabajo gratuito". Revista Internacional del Trabajo, vol.115, 6, (pp.701-707).

MOGIN BARQUÍN, M.T. (1997). «Provisión privada-social y gestión mixta de servicios públicos». Revista de Debate sobre Economía Pública Social y Cooperativa , 26. (pp. 11-22).

RENES, V., ALFARO, E. y RICCIARDELLI, O.(1994): El volunta riado social. Plan de formación de animadores. Bloque I. Madrid: CES.

SALINAS RAMOS, $F$ (1997). «El estado de bienestar y las organizaciones voluntarias» en VVAA.: ¿Trabajo voluntario 0 participación? Elementos para una sociología del Tercer Sector. Madrid: Tecnos (pp. 122-133).

VIDAL, I (1997):«España: Una nueva economía social aún poco conocida y reconocida" en VVAA: Inserción y nueva Economía Social (pp. 236-250). Madrid: CIRIEC.

VVAA (1998). Cambios en el trabajo y futuro del Derecho del Trabajo en Europa" Informe (Versión provisional).Grupo de expertos creado por la DG.V de la Comisión Europea. Luxemburgo. Oficina de Publicaciones de la Unión Europea.

VVAA (1998). Conclusiones de la Presidencia del Consejo Europeo Extraordinario sobre el empleo celebrado en Luxemburgo los días 20 y 21 de noviembre de 1997. Luxemburgo: Oficina de Publicaciones de la Unión Europea. 